\title{
A New Monte Carlo Model for Predicting the Mechanical Properties of Fiber Yarns
}

\author{
Xiaoding $\mathrm{Wei}^{1}$, Matthew Ford ${ }^{1}$, Rafael A. Soler-Crespo ${ }^{1}$, Horacio D. Espinosa ${ }^{1 *}$
}

${ }^{1}$ Department of Mechanical Engineering, Northwestern University

2145 Sheridan Road, Evanston, IL 60208-3111, USA

*corresponding author: espinosa@northwestern.edu

\begin{abstract}
Understanding the complicated failure mechanisms of hierarchical composites such as fiber yarns is essential for advanced materials design. In this study, we developed a new Monte Carlo model for predicting the mechanical properties of fiber yarns that includes statistical variation in fiber strength. Furthermore, a statistical shear load transfer law based on the shear lag analysis was derived and implemented to simulate the interactions between adjacent fibers and provide a more accurate tensile stress distribution along the overlap distance. Simulations on two types of yarns, made from different raw materials and based on distinct processing approaches, predict yarn strength values that compare favorably with experimental measurements. Furthermore, the model identified very distinct dominant failure mechanisms for the two materials, providing important insights into design features that can improve yarn strength.
\end{abstract}

Keywords: Monte Carlo, Hierarchical composites, Fiber yarns, Weibull statistics, Shear load transfer, Fiber rupture 


\section{Introduction}

Since their discovery in 1991, carbon nanotubes (CNTs) have attracted considerable attention in the mechanics community for their superior strength (up to $100 \mathrm{GPa}$ ) and elastic modulus (up to $1 \mathrm{TPa}$ ) (Sumio Iijima, 1991; M. Zhang et al., 2004). These outstanding mechanical properties make CNTs the ideal building blocks for macroscopic composites that require light weight and high mechanical performance for use in aerospace and automotive applications. One promising approach to scale up the mechanical properties of CNTs is to spin them into macroscopic yarns. Two main techniques have been developed to make macroscopic yarns, for instance: i) dry spinning of CNT yarns by drawing and twisting from CNT arrays, aerogels, or mats (V. DenisLutard et al., 2010; K. Koziol et al., 2007; J. Min et al., 2012; Mohammad Naraghi et al., 2010; Seongwoo Ryu et al., 2011; C.D. Tran et al., 2009; M. Zhang et al., 2004; S. Zhang et al., 2008); and ii) wet spinning of CNT yarns by drawing and twisting from CNT sources embedded in chemical solutions (Y.L. Li et al., 2004; X. Zhang et al., 2007). Similar to the micromechanics in staggered hierarchical biological composites, such as nacre shell (F Barthelat et al., 2007; Marc André Meyers et al., 2008), collagen fibril (Peter Fratzl et al., 1998) and spider silk (Sinan Keten et al., 2010), the load in CNT yarns is transferred over the overlap distance between fibrils via shear stresses at the interfaces. The nanoscale building blocks have two distinct failure modes - fibril rupture and interface sliding - that can eventually cause the failure of macroscopic yarns. Which of the two modes will dominate the composite failure depends on the critical dimensions and the mechanical properties of the building blocks (Benny Bar-On and $\mathrm{H}$ Daniel Wagner, 2013; Matthew R Begley et al., 2012; Hila Klein Selle et al., 2015; Haimin Yao et al., 2013). For yarns consisting of bare CNTs, the weak van der Waals interactions 
between carbon atoms cannot offer an interface strong enough to hold the integrity of yarns at a high load. Researchers have developed various techniques to improve the load transfer ability of the inter-fibril interfaces via either non-covalent bonds between functional chemistries on the CNTs surfaces or covalent bonds formed between crosslinked tubes (T Filleter and HD Espinosa, 2013; A Kis et al., 2004; Weibang Lu et al., 2012). Despite these efforts, the strengths of CNT yarns reported to date still fall short of the strength of individual CNTs. Identifying the primary cause of the yarn failure among the two competing failure modes - fibril rupture and interface sliding - is critical to advance this research.

Theoretical models have been explored to attempt to understand the predominant mechanisms of yarn failure. The first yarn model proposed by Daniels (HE Daniels, 1945) treated the yarn as a bundle of parallel fibers that were evenly clamped at each end and had no interactions between each other. These fibers had the same length and crosssectional area; however, the fiber strength distribution followed a Weibull probability function, i.e., the failure probability for a fiber under an applied stress $\sigma$ is given by

$$
P(\sigma)=1-\exp \left[-\frac{L_{b}}{L_{0}}\left(\frac{\sigma}{\sigma_{0}}\right)^{m}\right]
$$

where $L_{0}$ is the reference fiber length, $L_{b}$ is the fiber length, $\sigma_{0}$ is the scale factor, and $m$ is the shape factor. Daniels showed that when the load redistributes equally on intact fibers, the strength of the yarn asymptotically approaches a Gaussian distribution. Other models extended this approach to include more geometric factors. The continuum model developed by Hearle investigated the effects of fiber length, twisting angle, and fiber 
migration on the yarn mechanical properties (John WS Hearle et al., 1969). Hearle's model used a static friction law to simulate the interaction between fibers. Thus, statistics of the fiber strength and evolution of the microstructures, such as load redistribution after fiber breaks, are absent in Hearle's model. Porwal et al. (Pankaj K Porwal et al., 2006) extended Daniels' model and developed a Monte Carlo model to predict the statistical strength of a twisted fiber yarn using a twist-modified equal load sharing (TM-ELS) rule. Porwal's model takes into account the statistical variation of fiber strength and simulates fiber break initiation and progression for a yarn under tension. Later, with some modifications to the load sharing rule, this model was adopted to predict the mechanical strength of CNT yarns (IJ Beyerlein et al., 2009; Pankaj K Porwal et al., 2007). These existing models simplify interactions at the interfaces as a static friction law that requires a transverse pressure on fibers to cause load transfer. Furthermore, the friction law implies that the interactions between fibers can be enhanced linearly when the overlap distance increases, which is inconsistent with the experimental observation where the load transfer capability tends to saturate at some overlap distance (Xiaoding Wei et al., 2012). Therefore, the friction law is an oversimplification of the interfaces and may not accurately simulate the load distribution on fibers along the overlap distance.

In this study, we developed a new Monte Carlo model for fiber yarns in which the history of stochastic fibril rupture and interface sliding in macroscopic yarns is simulated and their effects on the mechanical properties of the fiber composites investigated. The interface is modeled as a soft thin layer that undergoes only shear deformation. The mechanical properties of the building blocks, fibril statistical strength and interface shear strength, are inputted from nanoscale experiments (T Filleter et al., 2011; T Filleter and 
HD Espinosa, 2013; Mohammad Naraghi et al., 2013; Mohammad Naraghi et al., 2010).

Case studies on two types of CNT yarns were performed using this model, and distinct bottlenecks for the mechanical performance of both types of yarns were identified.

\section{Model development}

\subsection{Yarn geometry and model discretization}

A twisted fiber yarn consisting of fibers in a hexagonal close-packing structure was assumed in our stochastic Monte Carlo model (Figure 1). Axial positions of individual fibers were randomly distributed to account for a random distribution of overlap lengths. Each fiber is discretized into a series of 1-D elements along the fiber axis, thus the normal stress in a fiber is uniform in the thickness dimension and only varies as a function of axial position.

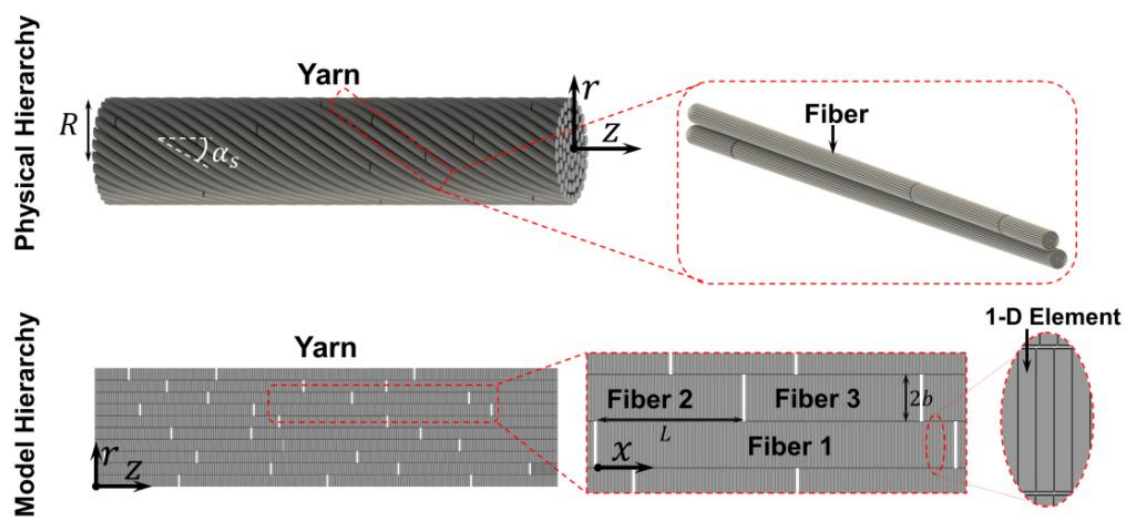

Figure 1. Schematics of idealized yarns depicting (left to right): the cross-sectional view of the yarn model, randomly distributed fibers (in this study, a fiber refers to a bundle consisting of 60 to 100 double-walled carbon nanotubes), and a fiber discretized by 1-D elements. (top) Hierarchical structure of a 3-D ideally twisted yarn consisting of hexagonal close-packed discontinuous fibers; (bottom) Yarn hierarchy in the Monte Carlo model (section view) reported here.

In a twisted yarn, it is necessary to transform fiber strains between the individual fiber axis and yarn axis along which the external load is applied. As noted by Hearle et al. 
(John WS Hearle et al., 1969), the relationship between the applied yarn strain $\varepsilon_{y}$ and the fiber strain $\varepsilon_{f}$, at radial position $r$, is given by

$$
\varepsilon_{f}(r)=\varepsilon_{y}\left(\cos ^{2} \alpha_{r}-v_{T} \sin ^{2} \alpha_{r}\right), \text { where } \alpha_{r}=\tan ^{-1}\left(\frac{r}{R} \tan \alpha_{s}\right)
$$

where $R$ is the yarn radius, $\alpha_{s}$ is the twist angle at the yarn surface, $\alpha_{r}$ is the twist angle of the fiber at radial position $r$, and $v_{T}$ is the Poisson's ratio of the yarn.

\subsection{Interface properties and load transfer simulation}

Unlike the static friction law in previous models (IJ Beyerlein et al., 2009; Pankaj K Porwal et al., 2006; Pankaj K Porwal et al., 2007), the load transfer between fibers in the current model is through interfaces that have well-defined mechanical properties, i.e., shear modulus $G$ and shear strength $\tau_{f}$. Note, however, that load transfer may not occur within all pairs of adjacent fibers. We therefore implemented an algorithm in our model to discriminate "effective" and "ineffective" contacts between two adjacent fibers based on their relative positions according to the following rules: 1) If one end of a fiber lies between the two ends of the adjacent fiber, the contact is defined as "effective", meaning that load can be effectively transferred between the two adjacent fibers through their interface (for example, the contact between fibers 1 and 2 in Figure 1). For an "effective" contact, the elastic solution for the shear lag model was used to calculate the maximum tensile stress distribution in each fiber, along the overlap region $L$, that can be transferred via the interface (Xiaoding Wei et al., 2012): 


$$
\sigma(x)=\frac{2 \tau_{f}}{b \lambda} \frac{1}{\cosh \left(\frac{\lambda L}{2}\right)} \sinh \left(\frac{\lambda x}{2}\right) \cosh \left(\frac{\lambda(L-x)}{2}\right),
$$

where $\lambda=\sqrt{2 G /(E b h)}, E$ is the fiber elastic modulus, $G$ is the interface shear modulus, $\tau_{f}$ is the interfacial shear strength, $h$ is the interface thickness. The equivalent fiber thickness is $2 b=A /(n w)$, in which $A$ is the cross-sectional area, $w$ is the contact width, and $n$ is the total number of effective contacts for a fiber with its nearest-neighbors (Xiaoding Wei et al., 2012). 2) If a fiber is shorter than the adjacent fiber, and both ends of the first fiber are completely enclosed by the adjacent fiber (for example, the contact between fibers 1 and 3 in Figure 1), the contact is defined as "ineffective." The load transfer through an "ineffective" contact is neglected in the present study for the following reasons: First, the main mechanism for a macroscopic yarn to carry external loads is the load transfer between two fibers that are in effective contact. In contrast, when a long fiber fully encloses a short fiber to form an ineffective contact, the load on the long fiber is just redistributed within the contact by the short fiber. This stress redistribution within an ineffective contact pair does not affect the overall load carrying capability of the macroscopic yarn significantly. Second, whether a contact is effective or not, a relative axial displacement between two fibers is essential for the load transfer or redistribution. In an effective contact, two fibers tend to slide relative to one another. By contrast, having a relative axial displacement is more difficult for two fibers in an ineffective contact. Thus, for the same axial contact length, the stress distribution is affected more by the effective contact than by the ineffective contact. In the investigated yarns, since the fibers are hexagonally close-packed, each fiber has 6 contacts with its 
neighbors. Therefore, even if there is one ineffective contact out of the 6 pairs, the load distribution in the fiber is still dominated by the load transfer through the remaining effective contacts.

Note that although the tensile stress distribution solution given by Equation (3) is from the shear lag analysis for two parallel fibers in an effective contact, it can be extended to twisted fiber yarns by introducing pressure dependence to the interface properties (such as shear modulus $G$ and shear strength $\tau_{f}$ ). When a twisted yarn is under tension, a transversal pressure between fibers will develop in the cross-section, which will affect the interfacial shear properties. By performing atomistic simulations, one can obtain the relationship between the shear modulus $G$ (as well as the shear strength $\tau_{f}$ ) and the lateral pressure $P$ for fibers (e.g. CNT bundles in the present study) sliding relative to one another. With the inputs of pressure dependent shear modulus $G(P)$ and shear strength $\tau_{f}(P)$, the extended shear lag analysis will provide a more precise description of the stress distribution for twisted fibers than the static friction assumption in previous models (IJ Beyerlein et al., 2009; Pankaj K Porwal et al., 2006; Pankaj K Porwal et al., 2007).

In addition, since load is transferred between adjacent fibers through shear forces, reducing the density of nearest-neighbor fibers leads to a reduction in the load-bearing capacity of the material. We model distributed micro-porosity by decreasing the maximum shear stress between fibers by a multiplication factor of $(1-p)$, for a yarn with porosity $p$. 


\subsection{Fiber properties and fragmentation simulation}

The elastic shear lag solution in Equation (3) gives the maximum tensile stress the elements along the overlap distance can carry before the interface slides (i.e. the shear stress at the interface reaches $\tau_{f}$ ). However, the true maximum tensile stress that can be applied on the elements is limited by the fiber strength. This brings another important failure mode - fiber rupture - into the model. CNT strength is extremely sensitive to the inherent defects that arise from manufacturing processes. Experimental studies (AH Barber et al., 2005; Asa H Barber et al., 2005; Claude A Klein, 2007) have shown that the CNT strength can be described by the Weibull distribution function expressed in Equation (1) in which the scale factor (the strength at which approximately 63\% CNT samples fail) depends on gauge length. Therefore, to take into account the variation in fiber strength and define the initiation and progression of fiber fragmentation, a random strength value needs to be assigned to the individual element. Similar to the approach used in previous models (Pankaj K Porwal et al., 2006; Pankaj K Porwal et al., 2007), the element strength values are chosen from a reservoir of random numbers generated by the Weibull distribution function given by Equation (1) with $L_{b}$ replaced by the 1-D fiber element size, $L_{e}$. However, we note that this approach raises the element size dependence issue. For example, for an extremely small element size, the scale factor of the Weibull function might be even greater than the theoretical strength of the fiber $\sigma_{t h}$, which is unphysical. To avoid this issue, a minimum length scale $L_{\min }=-L_{0}\left(\frac{\sigma_{0}}{\sigma_{t h}}\right)^{m} \log 10^{-2}$ was used in the model to limit the scale factor in the Weibull

function that creates the element strength reservoir. Thus, $L_{\min }$ has the physical meaning 
of being the average distance between defects. It assures that only $1 \%$ of element strength values exceed the theoretical fiber strength so that the Weibull scaling will not significantly affect the fiber fragmentation progression.

\subsection{Load-sharing rule}

The maximum stress an element can carry should be the minimum of two values - the maximum stress that can be transferred through the interface before sliding or the element strength. During simulations, an increasing elongation was applied to the yarn in steps, and the strain value on individual fiber was determined according to Equation (2). Then, the stress was distributed on the elements of fibers assuming the fibers are linear elastic. In each step, the distributed stress was compared with the maximum allowable stress of each element to determine whether interface sliding or fiber rupture occurs. If rupture occurred, two new fibers were created and the stress at the fragmentation location was set to zero. The model re-evaluated the effectiveness of each new fiber with their nearest-neighbors and the stress was redistributed. The iteration stopped when no new fiber breaks occurred in the current step and equilibrium was satisfied at all cross sections. Then an increasing load was applied to the yarn in the next step. The yarn was deemed as having failed when no additional load could be equilibrated. It should be noted that a full description of load transfer within a yarn requires solving equilibrium and compatibility at each point simultaneously. In the current model, to save computation cost, the loadsharing rules, as implemented, only ensure force equilibrium. The plastic portion of the yarn deformation due to interface sliding was not specifically accounted for. Based on this simplification, the model was aimed to predict the ultimate stress where the yarn fails rather than describe the full stress-strain response. 


\subsection{Model convergence and mesh dependence}

The goal of this study was to develop a Monte Carlo model from the micro/nanomechanics of CNT building blocks to predict the average value and distribution of fiber yarn strength. Therefore, accuracy of the statistical results was evaluated carefully. Model results showed that the yarn strength distribution approaches a Gaussian distribution function as the simulation number is increased. The mean and standard deviation converged for more than 100 simulations. In addition, we found that the model result became mesh independent when the element size was less than $0.5 \lambda^{-1}$

where $\lambda=\sqrt{2 G /(E b h)}$. In this report, an element size of $0.2 \lambda^{-1}$ was used and 100 simulations were performed for each case to obtain the mean and standard deviation of the yarn strength.

\section{Model application}

To demonstrate the model's predictive capabilities, case studies were performed on two types of yarns fabricated from different CNT sources. The first type was manufactured by dry spinning from double-walled carbon nanotube (DWCNT) mats produced by MER Corporation and characterized at Northwestern University (denoted as "NU yarns") (Mohammad Naraghi et al., 2010). The second type were multi-walled carbon nanotube (MWCNT) yarns fabricated at Rice University using the wet-spinning method, here termed "Rice yarns" (Natnael Behabtu et al., 2013).

\subsection{Mechanical properties of building blocks for DWCNT yarns (NU yarns)}

Figure 2 shows the hierarchy of the NU yarn down to individual fibers (i.e. doublewalled carbon nanotube (DWCNT) bundles in this case). Each DWCNT bundle consists of tens of hexagonally closed-packed DWCNTs and bundle diameter ranges from 10-30 
nm. Individual DWCNTs have an outer diameter of $2.2 \mathrm{~nm}$. X-ray photoelectron spectroscopy (XPS) and Fourier transform infrared (FTIR) spectroscopy characterizations determined that an inherent polymer coating (approximately $2.5 \mathrm{~nm}$ thick) exists on the bundle surface (Mohammad Naraghi et al., 2013). The mechanical properties of individual DWCNT bundles and bundle-bundle interfaces were characterized through nanoscale experiments and atomistic computations. By pooling DWNT bundle strength data from tensile strength experimental results previously reported (T Filleter et al., 2011; Mohammad Naraghi et al., 2013; Mohammad Naraghi et al., 2010; Dimitry Papkov et al., 2012), Weibull analysis on individual DWCNT bundles (30 $\mathrm{nm}$ diameter and $5 \mu \mathrm{m}$ length in average) yielded a scale factor $\sigma_{0}=2.8 \mathrm{GPa}$ and a shape factor $m=2.2$ ( Figure 3a). Note that the stress is defined here as the load on the bundle divided by the crosssectional area of all DWCNTs within the bundle. This should be discriminated from the "true stress" that is defined as the load divided by the cross-sectional area of only the DWCNTs on the outer layer of the bundle. The interface properties were obtained from in-situ scanning electron microscope (SEM) shear experiments on pairs of parallel bundles (Naraghi, Bratzel et al. 2013). According to the shear lag model analysis, the applied stress that causes the bundle-bundle junction to slide is a function of the overlap length $L$ as:

$$
\sigma_{\text {junction }}=\frac{2 \tau_{f}}{b \lambda} \tanh \left(\frac{\lambda L}{2}\right) .
$$

Fitting the experimental shear results with Equation (4) revealed an effective shear modulus of $G=10 \mathrm{MPa}$ and shear strength $\tau_{f}=350 \mathrm{MPa}$ (see Figure $3 \mathrm{~b}$ ). 


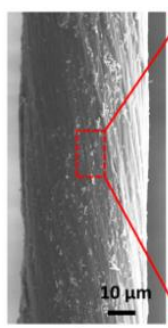

(a)

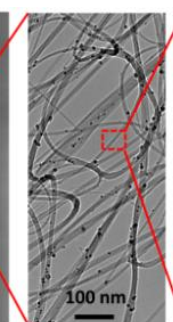

(b)

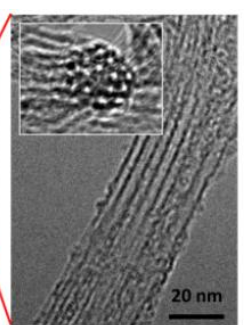

(c)

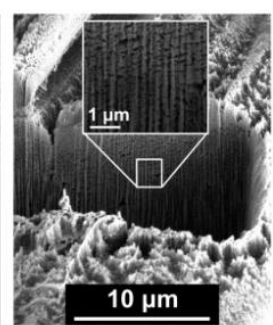

(d)

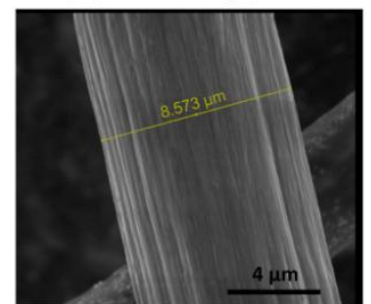

(e)

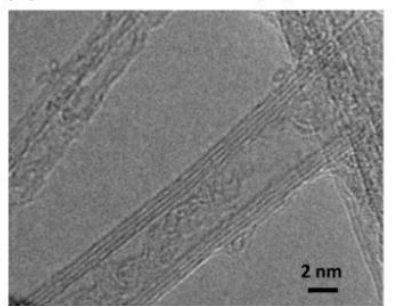

(f)

Figure 2. (top) From left to right: Images displaying the hierarchy of DWCNT yarns studied at Northwestern University. (a) SEM image of a NU yarn. (b) TEM image of interconnected DWCNT bundles picked from mats. (c) TEM image of individual DWCNT bundle with the inset image showing a cross section of an individual bundle. (d) A well was micromachined into a NU yarn using focused ion beam (FIB) to reveal the internal porosity. The inset highlights the porosity in the cross-section of the yarn. (bottom) From left to right: Images of the hierarchy of MWCNT yarns studied at Rice University (images are adapted from reference (Natnael Behabtu et al., 2013)). (d) SEM image of a Rice yarn. (e) TEM image of an individual MWCNT.

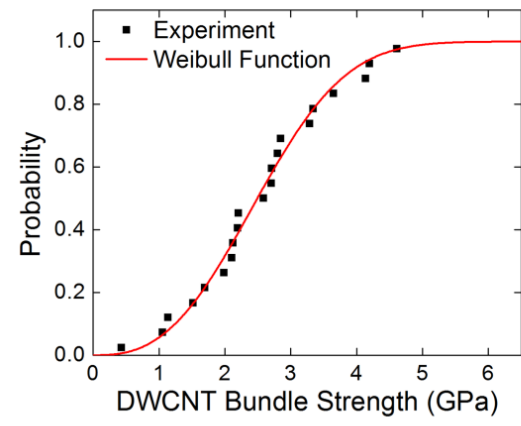

(a)

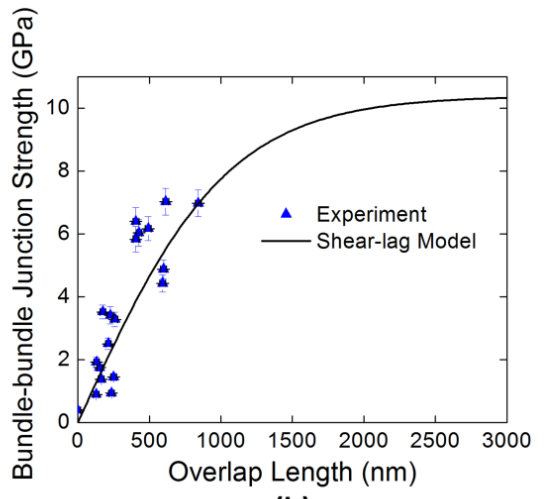

(b)

Figure 3. Mechanical properties of building blocks for NU yarns. (a) Weibull analysis on the individual DWCNT bundle strength compared to experimental values reported previously (T Filleter et al., 2011; Mohammad Naraghi et al., 2013; Mohammad Naraghi et al., 2010; Dimitry Papkov et al., 2012). (b) Fit of the experimental shear results for pairs of parallel DWCNT bundles reported previously (Naraghi, Bratzel et al. 2013) using the shear lag model. 


\subsection{Mechanical properties of building blocks for Rice yarns}

In Rice yarns, the constituent fibers are individual MWCNTs with an average length of 5 $\mu \mathrm{m}$ and an average diameter of $3.2 \mathrm{~nm}$. The mechanical properties of these MWCNTs were not characterized here, and therefore were approximated from independent studies. Tensile tests on MWCNTs performed by Yu et al. (Min-Feng Yu et al., 2000) yield a scale factor of $31.5 \mathrm{GPa}$, where the stress was defined by assuming only the outer shell of a MWCNT bears the load, and a shape factor of 2.4. By converting the stress value to an effective stress by accounting for the cross-sectional area of all of the shells, the scale factor used for the MWCNTs in Rice yarns was approximated as $14.8 \mathrm{GPa}$, while the shape factor remained the same. The interface properties between MWCNTs were estimated from the in-situ SEM shear experiments on pairs of parallel bare MWCNTs (Xiaoding Wei et al., 2012). Adopted from reference (Xiaoding Wei et al., 2012), the interfaces between MWCNTs in Rice yarns were assumed to have a shear strength of 60 $\mathrm{MPa}$ and a shear stiffness of $100 \mathrm{MPa}$. Note that molecular dynamics and density functional theory calculations suggest that the shear interaction between CNTs highly depends on the tube chirality and vacancy defects (Jeffrey T Paci et al., 2014; Xiaohua Zhang and Qingwen Li, 2009). The shear strength between a pair of chiral tubes (i.e., a turbostratic stacking instead of $\mathrm{AB}$ stacking of the shells in contact) might be as low as 0.24 MPa. On the other hand, presence of vacancy defects in tubes will increase the shear strength. To have a better estimation of the interface properties for Rice yarns, shear experiments on the constituents in those yarns are needed. In this study, the interface

properties reported in reference (Xiaoding Wei et al., 2012) are used as a first approximation. 
The properties of fibers and interfaces for NU and Rice yarns are summarized in Table 1. The theoretical strength of $30 \mathrm{~nm}$ diameter DWCNT bundles in NU yarns was estimated by assuming that the outer shells of the DWCNTs on the outer layer of the bundle fail at $100 \mathrm{GPa}$, the theoretical strength of a single shell of CNT (Bei Peng et al., 2008). Similarly, the theoretical strength of a MWCNT in Rice yarns was estimated by assuming that the outer shell of the MWCNT fails at $100 \mathrm{GPa}$.

Table 1. Mechanical properties of fibers and interfaces for NU and Rice yarns.

\begin{tabular}{|c|c|c|c|c|c|c|c|}
\hline & Fibers & $\begin{array}{c}\text { Fiber } \\
\text { diameter } \\
(\mathrm{nm})\end{array}$ & $\begin{array}{c}\text { Fiber } \\
\text { length, } \\
L_{b}(\mu \mathrm{m})\end{array}$ & $E(\mathrm{GPa})$ & $\sigma_{0}(\mathrm{GPa})$ & $m$ & $\begin{array}{c}\sigma_{t h} \\
(\mathrm{GPa})\end{array}$ \\
\hline $\begin{array}{c}\mathrm{NU} \\
\text { yarns }\end{array}$ & $\begin{array}{c}\text { DWCNT } \\
\text { bundles }\end{array}$ & $10-30$ & $\geq 5$ & 60 & 2.9 & 2.7 & 20.2 \\
\hline $\begin{array}{c}\text { Rice } \\
\text { yarns }\end{array}$ & MWCNTs & $2-6$ & 5 & 400 & 14.8 & 2.4 & 42.3 \\
\hline \hline $\begin{array}{c}\text { NU } \\
\text { yarns }\end{array}$ & \multicolumn{2}{|c|}{$\begin{array}{c}\text { Interface } \\
\text { thickness (nm) }\end{array}$} & $\begin{array}{c}\text { Effective interface } \\
\text { shear modulus (MPa) }\end{array}$ & \multicolumn{2}{|c|}{$\begin{array}{c}\text { Interface shear strength } \\
(\mathrm{MPa})\end{array}$} \\
\hline $\begin{array}{c}\text { Rice } \\
\text { yarns }\end{array}$ & 2.5 & \multicolumn{2}{|c|}{10} & \multicolumn{3}{|c|}{350} \\
\hline
\end{tabular}

\subsection{Model verification by coarse-grained molecular dynamics}

In order to verify the Monte Carlo modeling algorithm proposed in this study, a coarsegrained molecular dynamics simulation was performed on a small benchmark yarn, and the results were compared with those predicted by the Monte Carlo model on the same system. The benchmark yarn is $25 \mu \mathrm{m}$ long and $10 \mathrm{~nm}$ in diameter, and contains 95 $(10,10)-(16,16)$ DWCNTs in total (see the schematic in Figure 4a). The yarn crosssection is made of 19 tubes in hexagonal close packing, and four randomly distributed breaks determined five tubes in the longitudinal direction (i.e. the average tube length is 5 $\mu \mathrm{m})$. The bead-spring coarse-grained model developed by Cranford et al. for DWCNTs 
was used to perform a tensile test on the benchmark yarn (Steven W Cranford and Markus J Buehler, 2010). This coarse-grained model has been implemented in a variety of mesoscale models and has been proven to be a valid and efficient approach to simulate different configurations of CNT yarns and bundles (Graham H Bratzel et al., 2010; Steven Cranford et al., 2010; Reza Mirzaeifar et al., 2015; Mohammad Naraghi et al., 2013). In the coarse-grained model, the total energy of the system is expressed as:

$$
E_{\text {system }}=E_{b}+E_{\theta}+E_{\text {pairs }}
$$

where $E_{b}, E_{\theta}$ and $E_{\text {pairs }}$ represent the energy stored in chemical bonds due to axial stretching, bending and due to weak interactions, respectively. These energy contributions are represented, respectively, by:

$$
\begin{gathered}
E_{b}(r)=\frac{k_{b}}{2}\left(a-a_{0}\right)^{2} \\
E_{\theta}(\theta)=\frac{k_{\theta}}{2}\left(\theta-\theta_{0}\right)^{2} \\
E_{\text {pairs }}(d)=4 \varepsilon\left[\left(\frac{\delta}{d}\right)^{12}-\left(\frac{\delta}{d}\right)^{6}\right],
\end{gathered}
$$

in which $a$ is the bond length between two connected beads, $\theta$ is the bond angle, and $d$ is the distance between two non-bonded beads. Calibrated through full-atomistic simulations, the parameters for $(10,10)-(16,16)$ DWCNTs were determined as: $k_{b}=2000$ kcal mol ${ }^{-1} \AA^{-2}$ is the tensile stiffness of bonds, $a_{0}=10 \AA$ is the equilibrium bond distance, $k_{\theta}=45000 \mathrm{kcal} \mathrm{mol}^{-1} \mathrm{rad}^{-2}$ is the bending stiffness related to equilibrium bond angles, $\theta_{0}$ 
$=180^{\circ}$ is the equilibrium bond angle, $\varepsilon=21.60 \mathrm{kcal} \mathrm{mol}^{-1}$ is the Lennard-Jones energy well depth at equilibrium, and $\delta=22.63 \AA$ is the Lennard-Jones distance parameter (Reza Mirzaeifar et al., 2015). In order to simulate tube rupture, the original harmonic potential function in Equation (6) was modified as

$$
E_{b}(r)=\left\{\begin{array}{c}
\frac{k_{b}}{2}\left(a-a_{0}\right)^{2}, \text { for } a<a_{c u t} \\
0, \text { for } a \geq a_{\text {cut }}
\end{array}\right.
$$

where $a_{\text {cut }}$ is the cutoff length defining bond rupture. Since the harmonic potential function describes a linear-elastic stress-strain relationship for tubes under tension, the cutoff length can be related to the bond strength $\sigma_{f}$ by:

$$
a_{\text {cut }}=\left(1+\frac{\sigma_{f}}{E}\right) a_{0},
$$

where $E=400 \mathrm{GPa}$ is the DWCNT elastic modulus (Mohammad Naraghi et al., 2013)

The coarse-grained model for the benchmark yarn consists of 474,905 beads and 474,791 bonds. Using the algorithm discussed in Section 2.3, a reservoir of 474,791 bond strength values was generated using the Weibull distribution function with the scale and shape factors for the tube strength in Rice yarns listed in Table 1 (see Figure 4b). Accordingly, the Monte Carlo model contains also 474,791 1-D elements and the same tube configurations as in the coarse-grained model. The strength values from the same reservoir were assigned to the elements.

The coarse-grained simulation was carried out using the molecular dynamics package LAMMPS with a time step of $10 \mathrm{fs}$ (Steve Plimpton, 1995). The yarn was first minimized 
using a conjugated gradient algorithm, and then relaxed to reach equilibrium using the isothermal-isobaric (NPT) ensemble for $5 \mathrm{~ns}$ at $300 \mathrm{~K}$. Then, the two ends of the yarn were displaced to apply a tensile test with a strain increment of $0.01 \%$ until the yarn failed due to tube-tube sliding. After each strain increment, the system was relaxed for 0.25 ns using the canonical (NVT) at $300 \mathrm{~K}$. For direct comparison, the same strain increment $(0.01 \%)$ was used in the Monte Carlo model until the yarn failed. The stress vs. strain curve predicted by the coarse-grained model is shown in Figure 4c. The maximum yarn strength (1.02 GPa) given by the coarse-grained model agrees well with the value predicted by the Monte Carlo model (1.16 GPa). Furthermore, the accumulated tube ruptures as a function of the applied stress were compared in Figure 4d. In the coarsegrained simulation, the first tube rupture occurred when the yarn stress reached $0.82 \mathrm{GPa}$. The earliest tube rupture was observed in the Monte Carlo simulation at a very similar stress level $(0.76 \mathrm{GPa})$. At peak stress, 113 tube ruptures were observed in the coarsegrained simulation, slightly higher than the 91 observed in the Monte Carlo simulation. This can be explained by the fact that the load transfer between "ineffective" contacts is neglected in the Monte Carlo model. Thus, using the coarse-grained model trained for DWCNTs, we have shown that the new Monte Carlo model developed herein can correctly and efficiently predict yarns' strength and provide valuable details about the fragmentation of constituent fibers. This justifies our application of the Monte Carlo model to predict the behavior of two realistic CNT yarns synthesized, characterized, and measured in two different groups in the following section. 
(a)

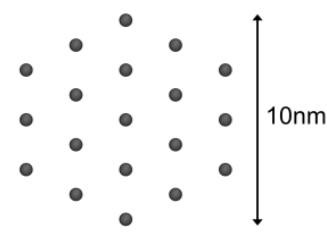

(c)

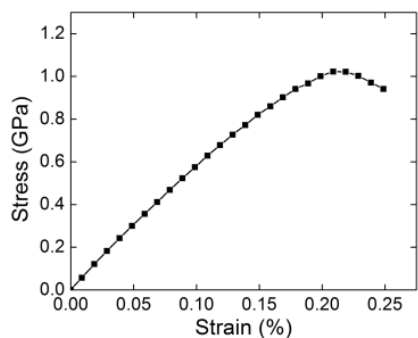

(b)

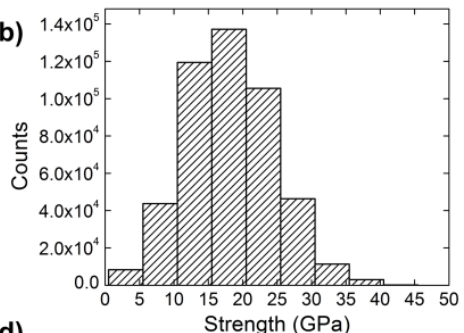

(d)

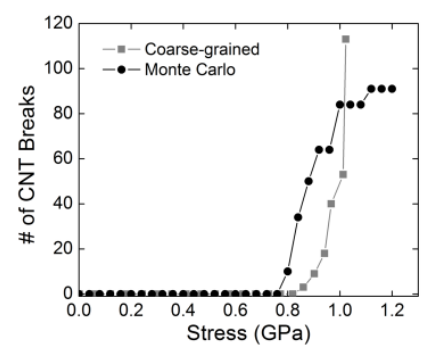

Figure 4. Verification of the Monte Carlo model by Coarse-grained simulations. (a) Front view (top) and side view (bottom) of the benchmark yarn. (b) Histogram of the strength values for tube bonds in coarse-grained model and 1-D elements in Monte Carlo model. (c) Stress vs. strain curve predicted by coarse-grained model. (d) Comparison between numbers of tube breakage predicted by coarse-grained model and Monte Carlo model.

\subsection{Modeling results and discussion}

Monte Carlo simulations were performed on both types of yarns listed in Table 1. Each yarn in the simulation was composed of 10 fibers along the yarn radius and 4 fibers along the yarn axis, for a total of 320 fibers. For each system, 100 Monte Carlo simulations were performed, and the average and standard deviation of the yarn strength were calculated. At the beginning of each simulation, the Weibull scale and shape factors listed in Table 1 were used to assign randomly distributed fiber element strengths using the algorithm discussed in Section 2.3. When an external load was applied on the yarn, the resulting stress distribution on fibers was determined using the load-sharing rule discussed in Section 2.4 with the mechanical parameters of the fibers and interfaces listed in Table 1 as the inputs. The external load kept increasing until no additional load could be equilibrated, and the maximum external load was recorded as the yarn strength. 
$\mathrm{X}$-ray and electron microscopy studies on Rice yarns revealed $5 \mu \mathrm{m}$ long MWCNTs aligned nearly perfectly along the axial direction, with no appreciable yarn porosity (Natnael Behabtu et al., 2013). Therefore, the actual microstructure in Rice yarns is very close to the idealized yarn structure assumption presented here. By contract, NU yarns have much more complicated microstructures that feature DWCNT bundle misalignment and entanglement as well as non-negligible yarn porosity ranging from $50 \%-80 \%$. In addition, the bundle geometries have noticeable variations. The majority of the bundle diameters are approximately $30 \mathrm{~nm}$, but they can be as small as $10 \mathrm{~nm}$. The distribution of bundle lengths is very difficult to precisely characterize. We speculate a range from 5 to $60 \mu \mathrm{m}$ based on SEM characterization of protruding bundles at the fracture surfaces of yarns. Nonetheless, it is very impractical to consider all these variations in the model. To provide useful insights for NU yarns, the model only selectively took into account critical factors. Simulations were performed first with the ideal helical twisted geometry to predict an upper bound of average yarn strength by neglecting complex features such as bundle misalignment and entanglement. As a baseline, $30 \mathrm{~nm}$ bundle diameter and $5 \mu \mathrm{m}$ bundle length were assumed in the model. The model then examined the effects of porosity and bundle size on the NU yarn strength. As shown in Figure 3b, the load transfer between bundles tends to saturate at an overlap length of $1.5 \mu \mathrm{m}$; therefore, the 5 $\mu \mathrm{m}$ bundle length assumption in the baseline simulations was appropriate given that our model does not account for bundle migration. For this reason, simulations performed on $60 \mu \mathrm{m}$ long bundles did not yield any appreciable difference.

Our model predicts the average strength of Rice yarns as $1.2 \pm 0.07 \mathrm{GPa}$. For the ideally twisted NU yarns consisting of $30 \mathrm{~nm}$ diameter and $5 \mu \mathrm{m}$ long bundles, the predicted 
yarn strength was $1.45 \pm 0.07 \mathrm{GPa}$. Shown in Figure 5a are the distributions of predicted yarn strength for NU and Rice yarns, suggesting that for both materials the strength distribution is virtually normal. Even though the predicted strength values for both yarns were similar, the model reveals different dominant failure mechanisms. As shown in Figure $5 b$, for the ideal NU yarn, the first bundle rupture occurred very early, at a small applied stress of $0.2 \mathrm{GPa}$, and the rupture rate accelerates gradually until the yarn fails. In the end, a total of approximately 3000 new bundles were generated due to rupture, almost 10 times the original number of bundles. This suggests that in the idealized NU yarns, the bundle strength is the limiting factor in the yarn performance. By contrast, MWCNT rupture in the Rice yarn occurred much later, at a high applied stress of 1.0 GPa (in the Weibull distribution tail for the MWCNT strength), and the total number of ruptured tubes only reached 12 when yarn failure occurred due to tube sliding and pull-out. This suggests that the interface strength is the limit for the Rice yarns' performance.

As discussed previously, the effective shear strength between bundles will be affected by yarn porosity. FIB and SEM characterizations on NU yarns indicated that the porosity ranges from $50 \%-80 \%$ (60\% on average). Figure $5 \mathrm{c}$ shows that the predicted NU yarn strength drops approximately linearly with yarn porosity up to $80 \%$, due to the previous assumption that the maximum shear strength between fibers decreases by a factor of (1-p). As the porosity increases, the effective interface shear strength weakens because individual bundles have fewer bundle-bundle contacts. Thus, the dominant failure mechanism becomes interface sliding. For example, the final number of bundle ruptures for $60 \%$ porous $\mathrm{NU}$ yarns is only 382 at an ultimate stress of $0.84 \mathrm{GPa}$ compared with 
1494 for the ideal helical NU yarns at an ultimate stress of $1.45 \mathrm{GPa}$; thus, a more porous yarn results in less effective bundle strengths within the yarns.

Finally, the effect of bundle diameter was examined. As noted by Filleter et al. (T Filleter et al., 2011), since the DWCNTs on the outer layer of the bundle carries the load, the effective bundle strength, which is defined by the load divided by the cross-sectional area of all the DWCNTs, is highly dependent on the bundle diameter. When the bundle diameter decreases, the ratio of the number of external to internal tubes increases, and the effective bundle strength will increase. Figure $5 \mathrm{~d}$ predicts that the average yarn strength could reach $4 \mathrm{GPa}$ if the bundle diameter were reduced to $10 \mathrm{~nm}$.

In summary, our model predictions are compared with experimental values in Table 2. The model prediction for Rice yarns $(1.2 \pm 0.07 \mathrm{GPa})$ agrees favorably with the experimental measurement $(1.0 \pm 0.2 \mathrm{GPa})$. For $\mathrm{NU}$ yarns with $60 \%$ porosity, the predicted yarn strength $(0.84 \pm 0.04 \mathrm{GPa})$ also agrees well with the experimental measurement $(0.95 \pm 0.4 \mathrm{GPa})$. The model predicted standard deviations for both materials that were smaller than the experimental values, especially for NU yarns. This is to be expected for this simplified model in which complexity such as fiber misalignment, entanglement, and waviness were not considered.

Table 2. Comparison between experimentally measured and model predicted strength for NU yarns and Rice yarns

\begin{tabular}{|c|c|c|c|c|}
\hline & $\begin{array}{c}\text { Surface twisting } \\
\text { angle, } \alpha_{s}\end{array}$ & $\begin{array}{c}\text { Porosity, } \\
p\end{array}$ & $\begin{array}{c}\text { Experimental yarn } \\
\text { strength }\end{array}$ & $\begin{array}{c}\text { Predicted yarn } \\
\text { strength }\end{array}$ \\
\hline $\begin{array}{c}\mathrm{NU} \\
\text { yarns }\end{array}$ & $\sim 15^{\circ}$ & $\sim 60 \%$ & $0.95 \pm 0.4 \mathrm{GPa}$ & $0.84 \pm 0.04 \mathrm{GPa}$ \\
\hline $\begin{array}{c}\text { Rice } \\
\text { yarns }\end{array}$ & $\sim 0^{\circ}$ & $\sim 0 \%$ & $1.0 \pm 0.2 \mathrm{GPa}$ & $1.2 \pm 0.07 \mathrm{GPa}$ \\
\hline
\end{tabular}




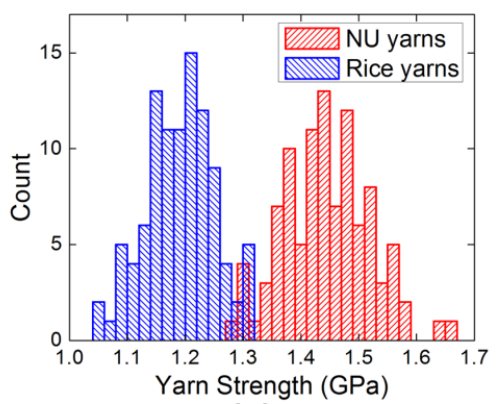

(a)

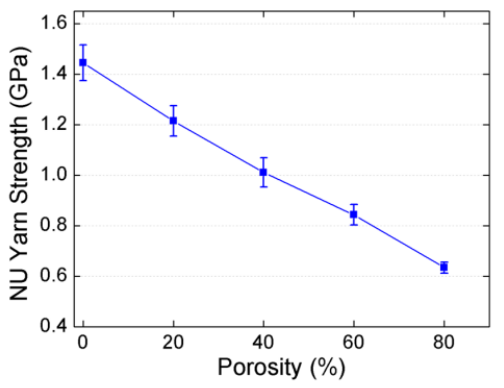

(c)

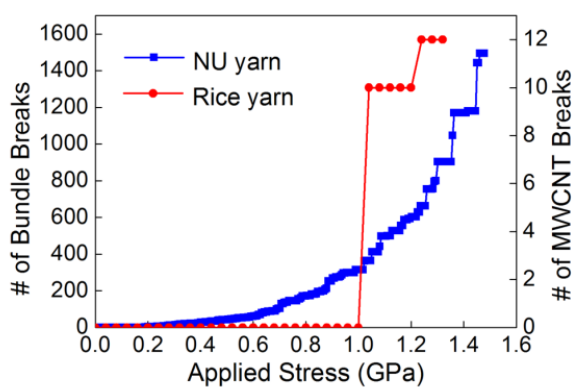

(b)

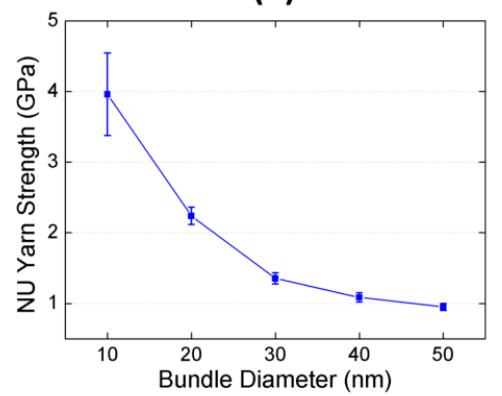

(d)

Figure 5. Model Predictions for NU and Rice yarns. (a) Distribution of Monte Carlo model-predicted yarn strengths for ideally twisted NU and Rice yarns. (b) Number of fiber ruptures as a function of the applied axial stress as predicted by the Monte Carlo model. (c) Predicted decrease in yarn strength due to the porosity in NU yarns. (d) Predicted yarn strength as a function of bundle diameter for NU yarns.

\section{Concluding remarks}

In this study, we developed a Monte Carlo model for hierarchical fiber yarns from the understanding of the statistics of individual fiber strength and the shear load transfer between pairs of discrete fibers. Adopting the solution for shear stress distribution from the shear lag model, a new load transfer law was implemented in the Monte Carlo model. Simulations were performed on two types of yarns made of different CNT resources to validate the predictive capability of the model. The key inputs for the Monte Carlo model, that is, the mechanical properties of building blocks (fibers and interfaces) were obtained directly from tensile and shear experiments on fibers at the nanoscale. The model prediction agrees well with the experimental measurement. Furthermore, the model provides valuable insights into the future possible approaches that should be explored to 
improve the yarn strength. Yet, the approaches for both materials are quite different. For NU yarns, the key to improve the strength is to: 1) reduce the yarn porosity (i.e. make yarns more compact); and 2) increase the fiber (i.e., DWCNT bundle) strength. Processing has an impact on both these features. A number of processing avenues have been investigated to overcome these barriers but many challenges still remain (Allison M. Beese et al., 2014). In these processes, achieving well-controlled bundle alignment remains challenging. Progress in manufacturing approaches that enables the selfassembly of constituents, as it is common in nature, is highly demanded. For Rice yarns, the effective way to increase the mechanical strength is to improve the interface shear strength. These yarns are produced from liquid crystal so that excellent constituent alignment has been achieved but at the expense of limiting the possible cross-linking chemistries or polymer matrices during the spinning process. Also, we note that the dominant failure mechanisms (fibril rupture and interface sliding) can transition from one to another when measures are carried out to improve the limiting factors. For example, using a generalized tension-shear chain mode, Zhang et al. found that as the cross-link density increase, the CNT bundles undergo a transition in failure mode from CNT pullout to CNT breakage (Zuoqi Zhang et al., 2014).

On the modeling side, more comprehensive models that account the realistic complex architectures of some CNT yarns (such as the entanglement of CNT bundles and effect of twisting on the load transfer capability between adjacent tubes or bundles) need to be developed. A multiscale approach, which is based on the inputs from bottom up measurements of mechanical properties of constituents at different scales, appears possible according to recent advances in coarse-grained and continuum modeling. 


\section{Acknowledgements}

The authors acknowledge the support of ARO through MURI Award No. W911NF-09-10541 and NSF through DMREF Award No. 1235480. The authors are thankful to insightful discussions with Alexander Moravsky of MER Corporation. 


\section{References}

Bar-On, B., Wagner, H.D., 2013. Structural motifs and elastic properties of hierarchical biological tissues-a review. Journal of structural biology 183, 149-164.

Barber, A., et al., 2005. Stochastic strength of nanotubes: an appraisal of available data. Composites science and technology 65, 2380-2384.

Barber, A.H., et al., 2005. On the tensile strength distribution of multiwalled carbon nanotubes. Applied Physics Letters 87, 203106.

Barthelat, F., et al., 2007. On the mechanics of mother-of-pearl: a key feature in the material hierarchical structure. Journal of the Mechanics and Physics of Solids 55, 306337.

Beese, A.M., et al., 2014. Key Factors Limiting Carbon Nanotube Yarn Strength: Exploring Processing-Structure-Property Relationships. ACS nano 11, 11454-11466.

Begley, M.R., et al., 2012. Micromechanical models to guide the development of synthetic 'brick and mortar'composites. Journal of the Mechanics and Physics of Solids 60, 1545-1560.

Behabtu, N., et al., 2013. Strong, light, multifunctional fibers of carbon nanotubes with ultrahigh conductivity. Science 339, 182-186.

Beyerlein, I., et al., 2009. Scale and twist effects on the strength of nanostructured yarns and reinforced composites. Nanotechnology 20, 485702.

Bratzel, G.H., et al., 2010. Bioinspired noncovalently crosslinked "fuzzy" carbon nanotube bundles with superior toughness and strength. Journal of Materials Chemistry 20, 10465-10474.

Cranford, S., et al., 2010. A single degree of freedom 'lollipop'model for carbon nanotube bundle formation. Journal of the Mechanics and Physics of Solids 58, 409-427.

Cranford, S.W., Buehler, M.J., 2010. In silico assembly and nanomechanical characterization of carbon nanotube buckypaper. Nanotechnology 21, 265706.

Daniels, H., 1945. The statistical theory of the strength of bundles of threads. I. Proceedings of the Royal Society of London. Series A. Mathematical and Physical Sciences 183, 405-435.

Denis-Lutard, V., et al., 2010. New Wet Spinning Process for the Continuous Production of Polymer/Carbon Nanotubes Composite Fibers, Recent Advances in Textile Composites: Proceedings of the 10th International Conference on Textile Composites. DEStech Publications, Inc, p. 167.

Filleter, T., et al., 2011. Ultrahigh Strength and Stiffness in Cross - Linked Hierarchical Carbon Nanotube Bundles. Advanced Materials 23, 2855-2860.

Filleter, T., Espinosa, H., 2013. Multi-scale mechanical improvement produced in carbon nanotube fibers by irradiation cross-linking. Carbon 56, 1-11.

Fratzl, P., et al., 1998. Fibrillar structure and mechanical properties of collagen. Journal of structural biology 122, 119-122. 
Hearle, J.W., et al., 1969. Structural mechanics of fibers, yarns, and fabrics.

Iijima, S., 1991. Helical microtubules of graphitic carbon. nature 354, 56-58.

Keten, S., et al., 2010. Nanoconfinement controls stiffness, strength and mechanical toughness of [beta]-sheet crystals in silk. Nature materials 9, 359-367.

Kis, A., et al., 2004. Reinforcement of single-walled carbon nanotube bundles by intertube bridging. Nature materials 3, 153-157.

Klein, C.A., 2007. Characteristic tensile strength and Weibull shape parameter of carbon nanotubes. Journal of applied physics 101, 124909.

Koziol, K., et al., 2007. High-performance carbon nanotube fiber. Science 318, 18921895.

Li, Y.L., et al., 2004. Direct spinning of carbon nanotube fibers from chemical vapor deposition synthesis. Science 304, 276-278.

Lu, W., et al., 2012. State of the art of carbon nanotube fibers: opportunities and challenges. Advanced Materials 24, 1805-1833.

Meyers, M.A., et al., 2008. Mechanical strength of abalone nacre: role of the soft organic layer. Journal of the Mechanical behavior of biomedical materials 1, 76-85.

Min, J., et al., 2012. High Performance Carbon Nanotube Spun Yarns from a Crosslinked Network. Carbon 52, 520-527.

Mirzaeifar, R., et al., 2015. Mesoscale mechanics of twisting carbon nanotube yarns. Nanoscale 7, 5435-5445.

Naraghi, M., et al., 2013. Atomistic investigation of load transfer between DWNT bundles "Crosslinked" by PMMA oligomers. Advanced Functional Materials 23, 18831892.

Naraghi, M., et al., 2010. A Multiscale Study of High Performance Double-Walled Nanotube-Polymer Fibers. ACS Nano 4, 6463-6476.

Paci, J.T., et al., 2014. Shear and friction between carbon nanotubes in bundles and yarns. to be appear in Nano Letters.

Papkov, D., et al., 2012. Extraordinary improvement of the graphitic structure of continuous carbon nanofibers templated with double wall carbon nanotubes. ACS nano 7, 126-142.

Peng, B., et al., 2008. Measurements of near-ultimate strength for multiwalled carbon nanotubes and irradiation-induced crosslinking improvements. Nature nanotechnology 3, 626-631.

Plimpton, S., 1995. Fast parallel algorithms for short-range molecular dynamics. Journal of computational physics 117, 1-19.

Porwal, P.K., et al., 2006. Statistical strength of a twisted fiber bundle: an extension of Daniels equal-load-sharing parallel bundle theory. Journal of Mechanics of Materials and Structures 1, 1425-1447. 
Porwal, P.K., et al., 2007. Statistical strength of twisted fiber bundles with load sharing controlled by frictional length scales. Journal of Mechanics of materials and Structures 4, 773-791.

Ryu, S., et al., 2011. High - Strength Carbon Nanotube Fibers Fabricated by Infiltration and Curing of Mussel - Inspired Catecholamine Polymer. Advanced Materials 23, 19711975.

Selle, H.K., et al., 2015. Gelatin yarns inspired by tendons-Structural and mechanical perspectives. Materials Science and Engineering: C 47, 1-7.

Tran, C.D., et al., 2009. Improving the tensile strength of carbon nanotube spun yarns using a modified spinning process. Carbon 47, 2662-2670.

Wei, X., et al., 2012. Optimal length scales emerging from shear load transfer in natural materials: application to carbon-based nanocomposite design. ACS nano 6, 2333-2344.

Yao, H., et al., 2013. Cracks fail to intensify stress in nacreous composites. Composites Science and Technology 81, 24-29.

$\mathrm{Yu}$, M.-F., et al., 2000. Strength and breaking mechanism of multiwalled carbon nanotubes under tensile load. Science 287, 637-640.

Zhang, M., et al., 2004. Multifunctional carbon nanotube yarns by downsizing an ancient technology Science 306, 1358-1361.

Zhang, S., et al., 2008. Solid-state spun fibers and yarns from 1-mm long carbon nanotube forests synthesized by water-assisted chemical vapor deposition. Journal of Materials Science 43, 4356-4362.

Zhang, X., Li, Q., 2009. Enhancement of friction between carbon nanotubes: an efficient strategy to strengthen fibers. Acs Nano 4, 312-316.

Zhang, X., et al., 2007. Strong Carbon - Nanotube Fibers Spun from Long Carbon Nanotube Arrays. Small 3, 244-248.

Zhang, Z., et al., 2014. Ultra-strong collagen-mimic carbon nanotube bundles. Carbon 77, 1040-1053. 